\title{
Atividade antioxidante e conteúdo fenólico do resíduo agroindustrial da produção de vinho
}

\section{Antioxidant activity and phenolic content of agricultural by-products from wine production}

\author{
Ciriele Boeira Cataneo ${ }^{1}$; Vinícius Caliari²; Luciano Valdemiro Gonzaga ${ }^{3}$; \\ Eugênia Marta Kuskoski ${ }^{4}$; Roseane Fett ${ }^{5^{*}}$
}

\begin{abstract}
Resumo
Este trabalho propõe uma investigação do potencial antioxidante do resíduo no processamento de uvas das variedades COUDERC 13 e PINOT GRIS, ambas coletadas em Videira-SC, como fonte natural de polifenóis e outros agentes profiláticos (antioxidantes) para aplicação nas indústrias alimentícia e de fitoterápicos. A biomassa utilizada para obtenção dos extratos foi triturada e seca a diferentes temperaturas $\left(45^{\circ} \mathrm{C}, 60^{\circ} \mathrm{C} \mathrm{e} 80^{\circ} \mathrm{C}\right)$, utilizando como solvente acetona $80 \%$. Os compostos fenólicos foram avaliados quantitativamente pelo método colorimétrico de Folin-Ciocalteu e os resultados expressos em mg/100g equivalente de ácido gálico. Para a determinação de flavanóis totais foi aplicado o método DMACA (pdimetilaminocinmaldeido) e os resultados foram expressos em equivalente a $\mathrm{mg}$ de catequina/100g de amostra. A atividade antioxidante total (AAT) foi avaliada pelo método espectrofotométrico através da descoloração do radical ABTS • + (2,2-azinobis[3-etil-benzotiazolin-6-ácido sulfônico]) com persulfato de potássio, e pelo método DPPH (2,2- Difenil-1picrilhidrazil), que tem como base a redução da absorbância do radical DPPH· por antioxidantes. Os resultados foram expressos em TEAC (atividade antioxidante equivalente ao Trolox) e VCEAC (atividade antioxidante equivalente à vitamina $\mathrm{C}$ ). Observou-se uma melhor atividade antioxidante nos bagaços de uva PINOT GRIS, sendo que os extratos secos a $45 \mathrm{oC}$ foram os que apresentaram um teor mais elevado de compostos fenólicos e de flavonóis totais, assim como uma melhor atividade antioxidante para ambas as variedades de uvas. Estas informações se tornam úteis pela identificação dos compostos fenólicos, e para que este resíduo seja possivelmente utilizado como aditivo seguro na indústria de alimentos.
\end{abstract}

Palavras-chave: Atividade antioxidante, extratos de uva, compostos fenólicos

\begin{abstract}
The present work aimed to investigate the antioxidant properties of byproducts derived from wine production. The varieties of PINOT GRIS and COUDERC 13 grapes, collected in Videira-SC, were analyzed as natural sources of polyphenols and other prophylactic agents (antioxidants) for application in food and phytochemical industries. The biomass used for extracts was ground and dried in different
\end{abstract}

1 Bolsista IC PIBIC/CNPq - Farmácia e Bioquímica UFSC. Universidade Federal de Santa Catarina, Centro de Ciências Agrárias, Departamento de Ciência e Tecnologia de Alimentos: Rodovia Admar Gonzaga, 1346, Itacorubi, CEP:88034-001, Florianópolis - SC Fone: 04833315375 Fax 04833319943 e-mail: ciriele_cataneo@yahoo.com.br; rfett@cca.ufsc.br

2 Químico Industrial Epagri/SC

3 Curso de Química UFSC

4 Pesquisadora Dra do Departamento de Ciência e Tecnologia de Alimentos CCA/UFSC

5 Profa. Dra do Departamento de Ciência e Tecnologia de Alimentos do Centro de Ciências Agrárias da Universidade Federal de Santa Catarina.

* Autor para correspondência 
temperatures $\left(45^{\circ} \mathrm{C}, 60^{\circ} \mathrm{C}\right.$ e $\left.80^{\circ} \mathrm{C}\right)$, using acetone $80 \%$ as solvent. The phenolic compounds were quantitatively evaluated by the Folin-Ciocalteu method and the results expressed in $\mathrm{mg} / 100 \mathrm{~g}$ of Gallic acid. For the flavonoids analysis, it was applied DMACA (p-Dimethylaminocinnamaldehye) method and the results were expressed in equivalent $\mathrm{mg}$ of catechin $/ 100 \mathrm{~g}$ sample. The antioxidant activity was evaluated by spectrophotometric method through the discoloration of ABTS ${ }^{++}$radical (2,2'-azino-bis[3ethylbenzthiazoline-6-sulfonic acid]) with potassium persulphate and by DPPH method, which is based on the reduction of DPPH' radical absorbance by antioxidant substances; the results were expressed in TEAC (antioxidant activity equivalent to Trolox) and VCEAC (antioxidant activity equivalent to vitamin C). It was observed a better antioxidant activity on extracts obtained from PINOT GRIS grapes, in which the $45^{\circ} \mathrm{C}$ dried extracts presented a higher number of phenolic compounds and flavonoids, as well as a better antioxidant activity in both varieties. These information become useful for phenolic compounds identifying and for a possible using of these extracts as a secure additive in food industry.

Key words: Antioxidant activity, grape extracts, phenolic compounds

\section{Introdução}

O Brasil, por ser um país de grande atividade agrícola, é um dos que mais produzem resíduos agroindustriais e a busca de alternativas para utilização da matéria orgânica gerada vem crescendo dentro de vários centros de pesquisa. Produtores e indústrias da área vinícola enfrentam o problema de descarte da biomassa residual que embora seja biodegradável, necessita de um tempo mínimo para ser mineralizada, constituindo-se numa fonte de poluentes ambientais. Dados da indústria mostram que para $100 \mathrm{~L}$ de vinho branco produzido geram-se $31,7 \mathrm{~kg}$ de resíduos, dos quais $20 \mathrm{~kg}$ são de bagaço (CAMPOS, 2005). Esses resíduos agroindustriais contêm uma variedade de espécies biologicamente ativas que são desperdiçadas, muitos deles ricos em compostos polifenólicos. Estima-se que, após o processamento das indústrias vinícolas, cerca de 13\% do peso total das uvas são descartados (RENAUD; DE LORGERIL, 1992; TORRES et al., 2002). A produção de uvas no Brasil está localizada, principalmente, nas regiões Sul, Sudeste e Nordeste. Constitui-se em atividade consolidada, com importância sócio-econômica, principalmente nos Estados do Rio Grande do Sul e de Santa Catarina, os quais respondem por $97 \%$ da produção nacional de vinhos (EMPRESA BRASILEIRA DE PESQUISA AGROPECUÁRIA, 2006). As duas variedades de uva utilizadas foram COUDERC $13 \mathrm{e}$ PINOT GRIS, ambas coletadas no estado de Santa Catarina. A primeira caracteriza-se por ser uma variedade híbrida de maturação tardia, de boa produção e rústica, com capacidade de originar vinho branco de ótimo aspecto, pouco aroma e de baixa acidez. A variedade PINOT GRIS é uma das mais utilizadas para elaboração de vinhos tintos na Europa e dentre suas antocianinas, encontram-se a delfinidina, cianidina, petunidina e peonidina (MAZZA, 1995).

Os compostos ativos mais comumente encontrados em frutas e hortaliças são as substâncias fenólicas (RHODES, 1996), as quais são conhecidas como potentes antioxidantes e antagonistas naturais de patógenos. Estas substâncias encontram-se nos vegetais na forma livre ou ligadas a açúcares e proteínas. Como antioxidantes naturais, além de serem compostos alternativos com finalidade de evitar a deterioração oxidativa dos alimentos, também podem exercer um importante papel fisiológico, minimizando os danos oxidativos no organismo animal (BLOCK, 1992; SANT'ANA; MANCINI FILHO, 1999; MELO; GUERRA, 2002.). Há evidências de que os fenólicos encontrados em uvas e vinhos tintos podem inibir a oxidação in vitro da lipoprotéina humana de baixa-densidade (LDL) (FRANKEL; WATERHOUSE; TEISSEDRE, 1995), assim como é possível seu uso na prevenção de aterosclerose (KOVAC; PEKIC, 1991).

A maioria das substâncias fenólicas pode ser classificada em dois principais grupos: os ácidos carboxílicos fenólicos e os flavonóides, sendo os flavonóides derivados do 2-fenil-benzopireno e classificados como o grupo mais importante (BITSCH, 1996). Os principais subgrupos são as catequinas e 
proantocianidinas, as antocianidinas e os flavonóis ou flavonas (HERRMANN, 1994). O reconhecimento dos benefícios à saúde, por parte das catequinas e proantocianidanas, está levando ao uso de extratos de semente de uva como suplementação alimentar (LAPARRA; MICHAUD; MASQUELIER, 1979).

Muitos estudos têm demonstrado que os radicais livres são os principais causadores de diversas doenças degenerativas, tais como doenças cardiovasculares, neurológicas e algumas formas de câncer (PRIOR et al., 1998). Os antioxidantes presentes nas plantas podem atuar como agentes redutores, seqüestradores de radicais livres, inibidores de enzimas e como quelantes de metais (WANG; LIN, 2000) e a maioria de seus efeitos biologicamente ativos são derivados das funções antioxidantes (VELIOGLO et al., 1998). Recentemente, há um aumento no interesse em antioxidantes naturalmente encontrados em frutos para uso em fitoterápicos, a fim de substituí-los pelos antioxidantes sintéticos, os quais têm uso restrito devido a seus efeitos colaterais, tais como carcinogenicidade (ITO et al., 1983). Além disso, os antioxidantes naturais possuem a capacidade de melhorar a qualidade e a estabilidade dos alimentos, agirem como nutracêuticos e proporcionar, ainda, benefícios adicionais à saúde dos consumidores (ARUOMA, 1998; LAI; CHOU; CHAO, 2001).

O objetivo deste trabalho foi avaliar quantitativamente os compostos fenólicos e o potencial antioxidante do resíduo agroindustrial da produção de vinhos, de uvas das variedades COUDERC 13 e PINOT GRIS, ambas coletadas no estado de Santa Catarina, visando a possibilidade de utilização dos mesmos, como fonte de compostos de interesse às indústrias de fitoterápicos e de complementos alimentares.

\section{Materiais e Métodos}

Amostras

As amostras de bagaço de uva das variedades COUDERC 13 (híbridas americanas) e PINOT GRIS (Vitis vinifera) foram cedidas pela EPAGRI
(Empresa Catarinense de Pesquisa Agropecuária) de Videira, Estado de Santa Catarina. Com o objetivo de preservar as propriedades gerais dos bagaços, os exemplares foram acondicionados em caixas isotérmicas contendo gelo, sendo encaminhadas imediatamente para laboratório, onde foram armazenados à temperatura de $-18,0 \pm 0,2^{\circ} \mathrm{C}$ até uso.

\section{Matéria seca}

O conteúdo de matéria seca dos bagaços das variedades de uva (COUDERC 13 e PINOT GRIS) foi obtido em estufa a $105^{\circ} \mathrm{C}$, por $24 \mathrm{~h}$ (AUSTRALIAN OFFICIAL ANALYTICAL CHEMISTS, 2002). O extrato seco foi pesado e o rendimento foi calculado baseado no peso úmido das amostras. Os resultados foram usados para expressar o conteúdo de fenólicos totais e a atividade antioxidante por peso seco.

\section{Preparação dos extratos}

Para obtenção dos extratos, as amostras do bagaço de uva das variedades COUDERC 13 e PINOT GRIS foram submetidas a tratamento térmico à $80^{\circ} \mathrm{C}$, num período de $10 \mathrm{~min}$, para inativação enzimática (VALDERRAMA; MARANGONI; CLEMENTE, 2001). Posteriormente, estas foram levadas à secura, em estufa de ar circulante, a diferentes temperaturas $\left(45^{\circ} \mathrm{C}, 60^{\circ} \mathrm{C}\right.$ e $\left.80^{\circ} \mathrm{C}\right)$, por 4 horas. Após resfriamento em dessecador, as amostras foram trituradas (60 mesh) em moinho IKQ AK41. Os extratos foram, então, preparados em triplicata a partir do material seco $(4,0 \mathrm{~g})$, utilizando-se $50 \mathrm{~mL}$ de acetona $80 \%$ como solvente extrator, sob agitação mecânica ao abrigo total da luz, durante um período de duas horas. Os extratos foram filtrados em papel filtro Whatman $\mathrm{n}^{\mathrm{o}} 1$ e transferidos para balão volumétrico, sendo o volume final ajustado para $50 \mathrm{~mL}$ com acetona $80 \%$ ou concentrados sob gás nitrogênio.

Determinação do conteúdo de polifenóis totais (PT)

Os compostos fenólicos totais foram determinados, em triplicata, pelo método colorimétrico 
de Folin-Ciocalteu, com algumas modificações (BUDINI, TONELLI, GIROTTI, 1980) e os resultados expressos em equivalentes de ácido gálico (GAE, mg/100g).

\section{Determinação da atividade antioxidante pelo método ABTS}

O método ABTS utilizado foi descrito por RE et al. (1999). A absorbância foi medida em espectrofotômetro modelo Hewlett-Packard 8452 A, no tempo de 7 minutos após a adição da amostra.

A capacidade antioxidante total das amostras foi calculada em relação à atividade do Trolox e do ácido ascórbico nas mesmas condições. Os experimentos foram realizados em triplicata e os resultados expressos em mg/100g e $\mu \mathrm{mol} / \mathrm{g}$ de TEAC (atividade antioxidante equivalente ao trolox) (RICE-EVANS; MILLER; PAGANGA, 1996) e VCEAC (atividade antioxidante equivalente à vitamina $\mathrm{C}$ ) (KIM; JEONG; LEE, 2003).

\section{Método DPPH (2,2-Difenil-1-picrilhidrazilo)}

A capacidade antioxidante foi, também, determinada pela redução do radical estável DPPH através da ação dos antioxidantes presentes na amostra, de acordo com o método modificado por Kim, Jeong e Lee (2003). A medida de absorbância do radical, antes de adicionar a amostra $\left(\mathrm{A}_{0}\right)$ e depois de 30 minutos de reação $\left(A_{f}\right)$, foi realizada no comprimento de onda de $517 \mathrm{~nm}$. Os experimentos foram analisados em triplicata e os resultados expressos em TEAC, em $\mu \mathrm{Mol}$ TEAC/g de amostra.

\section{Determinação de flavanóis totais (FT)}

A determinação de flavanóis foi realizada aplicando-se o método DMACA ( $p$ dimetilaminocinmaldeído), descrito por Arnous, Makris e Kefalas (2002). A concentração total de flavanóis foi estimada conforme a curva de calibração, preparada com solução de catequina (1-
$16 \mathrm{mg}$ de catequina/L). Os experimentos foram realizados em triplicata e os resultados expressos em equivalente a mg catequina/100g de amostra.

\section{Análise estatística}

Todos os experimentos foram realizados em triplicata e todos os resultados apresentados como média \pm desvio padrão (DP). Foram realizadas análises de variância (ANOVA) e o teste de Tukey para identificar diferenças significativas entre as médias, usando o programa Statistica ${ }^{\circledR}$ 6.0. Diferenças entre as médias no nível de $5 \%(\mathrm{P}<0,05)$ foram consideradas significantes.

\section{Resultados e Discussão}

Efeito do tratamento térmico no conteúdo de fenólicos totais

Os compostos fenólicos totais, nos extratos de acetona $80 \%$, variaram no bagaço seco da uva COUDERC 13 entre 109,64 a 207,80 GAE mg/100g, e entre 370,17 e 420,61 mg GAE/100g nos extratos do bagaço seco de uva PINOT GRIS. Os compostos fenólicos totais no extrato acetônico apresentaram aumento significativo com o tratamento térmico a $45^{\circ} \mathrm{C}$. (Tabelas 1 e 2 ).

Estudos anteriores mostraram que há conversão de compostos fenólicos insolúveis em solúveis, após tratamento com calor; porém, o mesmo não consegue romper ligações covalentes dos compostos fenólicos (LEE et al.,2003).

O teor de flavanóis totais apresentou valores que variaram entre 53,88 e 106,22 mg/100g, no bagaço seco da uva COUDERC 13 e entre 271,32 e 346,21 $\mathrm{mg} / 100 \mathrm{~g}$, nos extratos da uva PINOT GRIS. Observou-se, igualmente, um aumento significativo do teor de flavanóis totais nos extratos acetônicos, com o tratamento térmico a $45^{\circ} \mathrm{C}$ (Tabelas 1 e 2 ). Negro, Tommasi e Miceli (2003) encontraram, para bagaços de uvas vermelhas da variedade NEGRO AMARO, valores médios de flavanóis de 3,94 $\pm 0,08$ $\mathrm{g} / 100 \mathrm{~g}$ peso seco. 
Tabela 1. Efeito do tratamento térmico no conteúdo de polifenóis totais (PT), flavanóis e atividade antioxidante (ABTS e DPPH) do bagaço da uva COUDERC 13 em acetona 80\% (media \pm DP, $n=3$ ) em base seca.

\begin{tabular}{cccccc}
\hline Amostras & $\begin{array}{c}\mathbf{P T}^{1} \\
(\mathbf{m g} / \mathbf{1 0 0 g})\end{array}$ & $\begin{array}{c}\text { Flavanóis }^{2} \\
(\mathbf{m g} / \mathbf{1 0 0 g})\end{array}$ & $\begin{array}{c}\text { ABTS } \\
\text { TEAC }^{3} \\
(\mu \mathbf{m o l} / \mathbf{g})\end{array}$ & $\begin{array}{c}\text { ABTS } \\
\mathbf{V C E A C}^{4} \\
(\mathbf{m g} / \mathbf{1 0 0 g})\end{array}$ & $\begin{array}{c}\text { DPPH } \\
\text { TEAC }^{5} \\
(\boldsymbol{\mu m o l} / \mathbf{g})\end{array}$ \\
\hline BUC & $171,67 \pm 3,10^{\mathrm{a}}$ & $81,55 \pm 3,82^{\mathrm{a}}$ & $80,96 \pm 6,42^{\mathrm{a}}$ & $912,68 \pm 13,34^{\mathrm{a}}$ & $191,49 \pm 3,15^{\mathrm{a}}$ \\
BUC 45C & $207,79 \pm 1,57^{\mathrm{b}}$ & $106,22 \pm 1,98^{\mathrm{b}}$ & $98,92 \pm 5,27^{\mathrm{a}}$ & $964,08 \pm 2,33^{\mathrm{b}}$ & $220,44 \pm 4,03^{\mathrm{b}}$ \\
BUC 60C & $115,23 \pm 5,09^{\mathrm{c}}$ & $70,33 \pm 1,25^{\mathrm{a}, \mathrm{c}}$ & $50,29 \pm 1,65^{\mathrm{c}}$ & $814,06 \pm 11,94^{\mathrm{c}}$ & $123,22 \pm 3,99^{\mathrm{c}}$ \\
BUC 80C & $109,64 \pm 1,05^{\mathrm{c}}$ & $53,88 \pm 1,49^{\mathrm{c}}$ & $42,69 \pm 3,96^{\mathrm{c}}$ & $735,21 \pm 9,30^{\mathrm{d}}$ & $116,02 \pm 3,23^{\mathrm{c}}$ \\
\hline
\end{tabular}

BUC: extrato do resíduo de uva Couderc 13 sem tratamento; BUC 45C: extrato do resíduo de uva Couderc 13 aquecido a $45^{\circ} \mathrm{C}$; $\mathrm{BUC} 60 \mathrm{C}$ : extrato do resíduo de uva Couderc 13 aquecido a $60^{\circ} \mathrm{C}$; BUC $80 \mathrm{C}$ : extrato do resíduo de uva Couderc 13 aquecido a $80^{\circ} \mathrm{C}$.

${ }^{1} \mathrm{PT}=$ Polifenóis Totais expressos em GAE $=$ equivalente ao ácido gálico $\mathrm{mg} / 100 \mathrm{~g} ;{ }^{2}$ Flavanóis expressos em $\mathrm{CE}=$ equivalente à catequina.; ${ }^{3}$ Atividade Antioxidante pelo método ABTS expressa em TEAC $=$ atividade antioxidante equivalente ao trolox em 7 min; ${ }^{4}$ Atividade Antioxidante pelo método ABTS expressa em VCEAC $=$ atividade antioxidante equivalente a Vitamina $\mathrm{C} ;{ }^{\mathbf{5}}$ Atividade Antioxidante pelo método DPPH expressa em TEAC = atividade antioxidante equivalente ao trolox em $30 \mathrm{~min}$. Letras diferentes na mesma coluna implicam diferença significativa $(P<0,05)$ entre as amostras pelo Teste de Tukey.

Tabela 2. Efeito do tratamento térmico no conteúdo de polifenóis totais (PT), flavanóis e atividade antioxidante (ABTS e DPPH) do bagaço da uva PINOT GRIS em acetona $80 \%$ (media \pm DP, $n=3$ ) em base seca.

\begin{tabular}{cccccc}
\hline Amostras & $\begin{array}{c}\mathbf{P T}^{1} \\
(\mathbf{m g} / \mathbf{1 0 0 g})\end{array}$ & $\begin{array}{c}\text { Flavanóis }^{2} \\
(\mathbf{m g} / \mathbf{1 0 0 g})\end{array}$ & $\begin{array}{c}\text { ABTS } \\
\text { TEAC }^{3} \\
(\mu \mathbf{m o l} / \mathbf{g})\end{array}$ & $\begin{array}{c}\text { ABTS } \\
\text { VCEAC }^{4} \\
(\mathbf{m g} / \mathbf{1 0 0 g})\end{array}$ & $\begin{array}{c}\text { DPPH } \\
\text { TEAC }^{5} \\
(\mu \mathbf{m o l} / \mathbf{g})\end{array}$ \\
\hline BUP & $375,68 \pm 5,34^{\mathrm{a}}$ & $289,32 \pm 2,33^{\mathrm{a}}$ & $359,19 \pm 6,76^{\mathrm{a}}$ & $1124,52 \pm 3,40^{\mathrm{a}}$ & $380,09 \pm 3,23^{\mathrm{a}}$ \\
BUP 45C & $420,61 \pm 1,92^{\mathrm{b}}$ & $346,21 \pm 2,86^{\mathrm{a}, \mathrm{b}}$ & $463,46 \pm 2,76^{\mathrm{b}}$ & $1484,63 \pm 5,28^{\mathrm{b}}$ & $483,31 \pm 4,65^{\mathrm{b}}$ \\
BUP 60C & $391,62 \pm 2,64^{\mathrm{a}, \mathrm{b}, \mathrm{c}}$ & $309,55 \pm 2,99^{\mathrm{a}, \mathrm{b}, \mathrm{c}}$ & $386,04 \pm 3,62^{\mathrm{a}, \mathrm{c}}$ & $1214,65 \pm 2,38^{\mathrm{c}}$ & $396,33 \pm 4,85^{\mathrm{a}, \mathrm{c}}$ \\
BUP 80C & $370,16 \pm 3,68^{\mathrm{a}, \mathrm{c}}$ & $271,32 \pm 1,96^{\mathrm{a}, \mathrm{c}}$ & $347,88 \pm 6,63^{\mathrm{a}, \mathrm{c}}$ & $1086,41 \pm 6,25^{\mathrm{a}}$ & $369,23 \pm 4,21^{\mathrm{a}, \mathrm{c}}$ \\
\hline
\end{tabular}

BUP: extrato do resíduo de uva Pjnot Gris sem tratamento; BUP 45C: extrato do resíduo de uva Pjnot Gris aquecido a $45^{\circ} \mathrm{C}$; $\mathrm{BUP}$ 60C: extrato do resíduo de uva Pjnot Gris aquecido a $60^{\circ} \mathrm{C}$; BUP 80: extrato do resíduo de uva Pjnot Gris aquecido a $80^{\circ} \mathrm{C}$.

${ }^{1} \mathrm{PT}=$ Polifenóis Totais expressos em GAE = equivalente ao ácido gálico $\mathrm{mg} / 100 \mathrm{~g} ;{ }^{2}$ Flavanóis expressos em $\mathrm{CE}=$ equivalente à catequina.; ${ }^{3}$ Atividade Antioxidante pelo método ABTS expressa em TEAC $=$ atividade antioxidante equivalente ao trolox em 7 min; ${ }^{4}$ Atividade Antioxidante pelo método ABTS expressa em VCEAC $=$ atividade antioxidante equivalente a Vitamina $\mathrm{C} ;{ }^{\mathbf{5}}$ Atividade Antioxidante pelo método DPPH expressa em TEAC = atividade antioxidante equivalente ao trolox em $30 \mathrm{~min}$. Letras diferentes na mesma coluna implicam diferença significativa $(P<0,05)$ entre as amostras pelo Teste de Tukey.

Pode-se considerar um teor bastante significativo de compostos fenólicos na amostra da uva PINOT GRIS quando comparada com estudos, como o realizado por Llobera e Cañellas (2006) com bagaço de uvas vermelhas da variedade Manto Negro (Vitis vinifera), onde foram encontrados valores de compostos fenólicos de 2,63 g GAE/100g.

Os resultados obtidos da capacidade antioxidante, determinadas pelo método ABTS, foram, em média, de 463,46 $\mu \mathrm{Mol} \mathrm{TEAC/g}$ na variedade PINOT GRIS, e de $98,92 \mu \mathrm{Mol} / \mathrm{g}$ para variedade COUDERC 13 , obtidos do bagaço seco a $45^{\circ} \mathrm{C}$ e medidos em 7 minutos de reação (Tabelas 1 e 2).

Com relação aos valores encontrados para a atividade antioxidante equivalente ao ácido ascórbico (VCEAC), houve variação entre mínimos e máximos de 735,21 e $964,08 \mathrm{mg} / 100 \mathrm{~g}$ VCEAC para a uva COUDERC 13 e 1086,41 e 1484,63 mg/100g VCEAC para a variedade PINOT GRIS e a média dos valores de VCEAC se correlaciona de forma 
positiva com a média dos valores de polifenóis totais para os extratos analisados $\left(r^{2}=0,9749\right.$ para uva COUDERC e $r^{2}=0,9878$, para uva PINOT GRIS).

As amostras de bagaço que foram submetidas à secagem a $45^{\circ} \mathrm{C}$ apresentaram maior conteúdo de compostos fenólicos, assim como maior valor TEAC determinado pelo método DPPH, conforme pode-se observar nas Figuras 1 e 2 . O bagaço seco a $45^{\circ} \mathrm{C}$ e o úmido apresentaram diferenças significativas a nível de $95 \%$ de confiança.

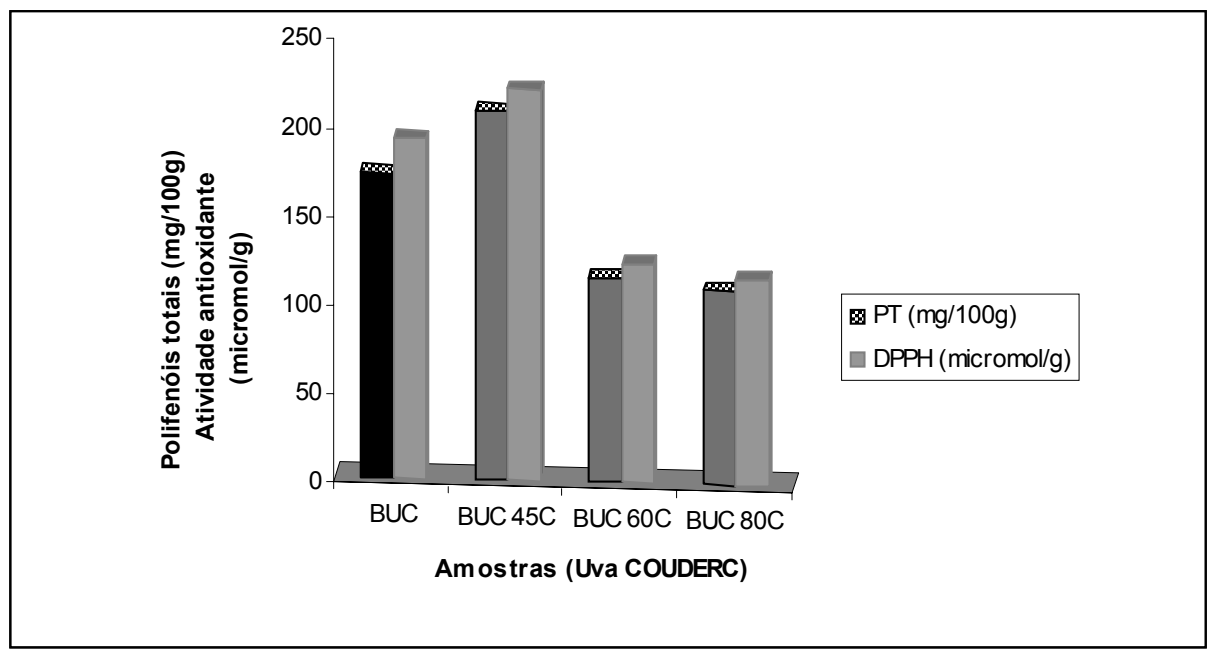

Figura 1. Polifenóis totais e atividade antioxidante (DPPH) dos extratos obtidos do bagaço úmido e seco a 45,60 e $80^{\circ} \mathrm{C}$ da variedade COUDERC 13.

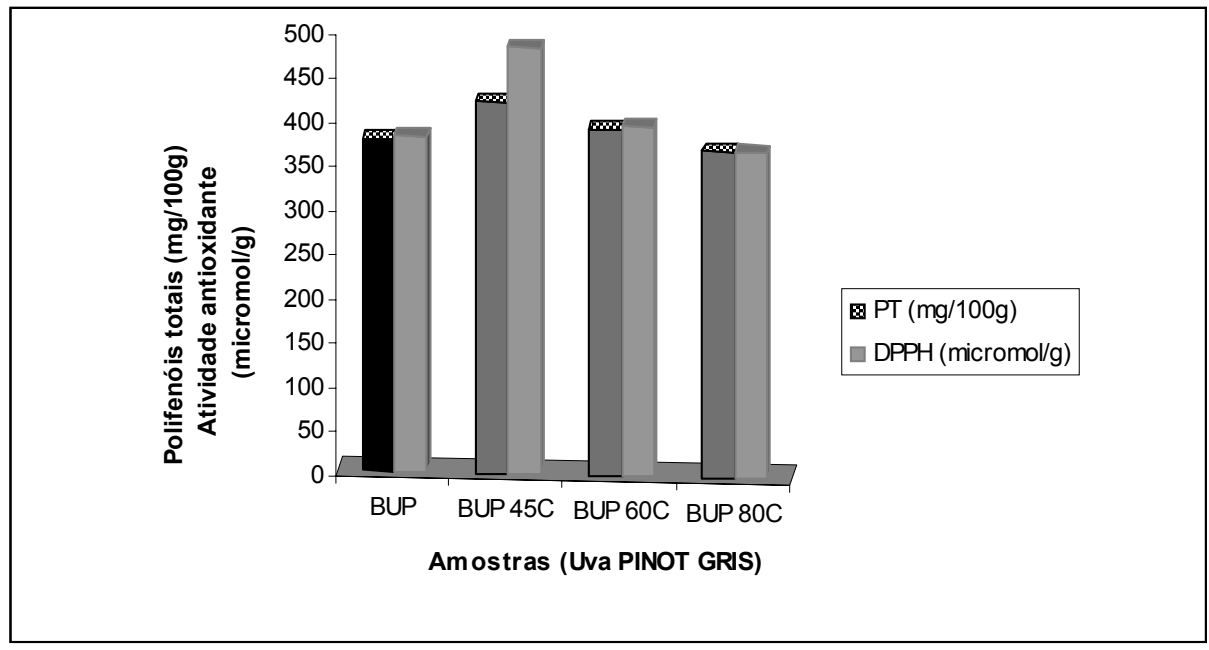

Figura 2. Polifenóis totais e atividade antioxidante (DPPH) dos extratos obtidos do bagaço úmido e seco a 45 , 60 e $80^{\circ} \mathrm{C}$ da variedade PINOT GRIS.

Vários estudos reportaram a relação entre conteúdo de fenólicos e atividade antioxidante. Velioglu et al., (1998) e verificaram uma forte relação entre ambos em frutas, legumes e produtos de grãos.

O coeficiente de correlação entre o conteúdo de polifenóis totais e a atividade antioxidante (método
ABTS) encontrado foi de 0,9896 e $0,9939(P<0,05)$, para as variedades COUDERC 13 e PINOT GRIS, respectivamente (Figuras 3 e 4). Os resultados obtidos indicam que o resíduo da produção de vinho é um produto com capacidade antioxidante significativa e estão correlacionados ao conteúdo de compostos fenólicos totais. 


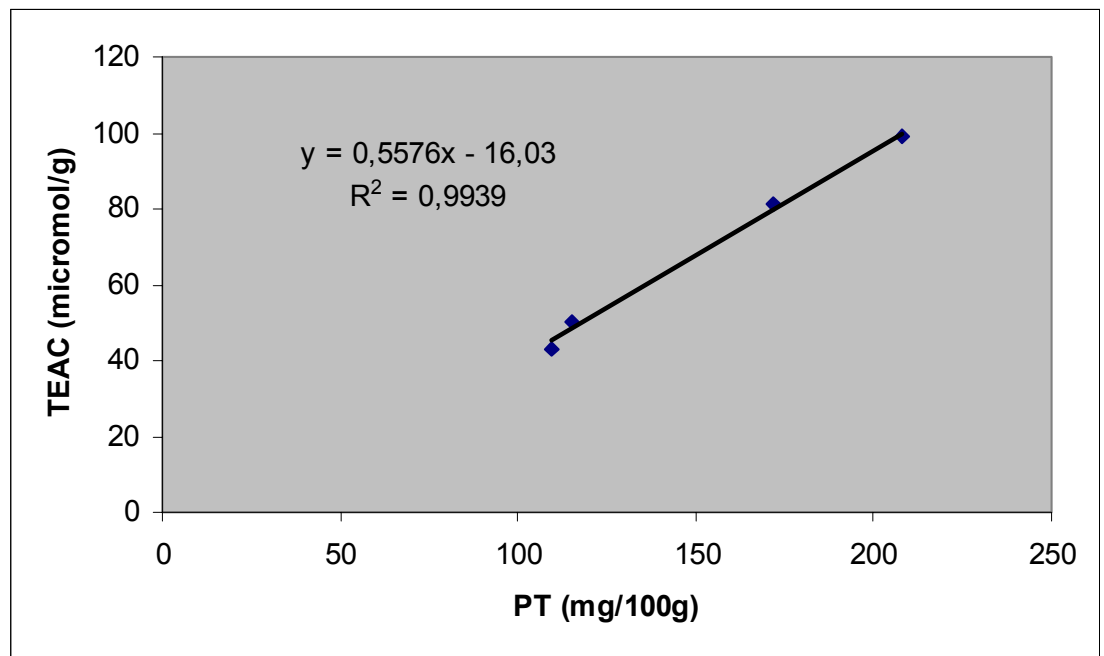

Figura 3. Correlação entre a atividade antioxidante equivalente ao Trolox (TEAC) e conteúdo de polifenóis totais (PT) dos extratos obtidos de uva COUDERC.

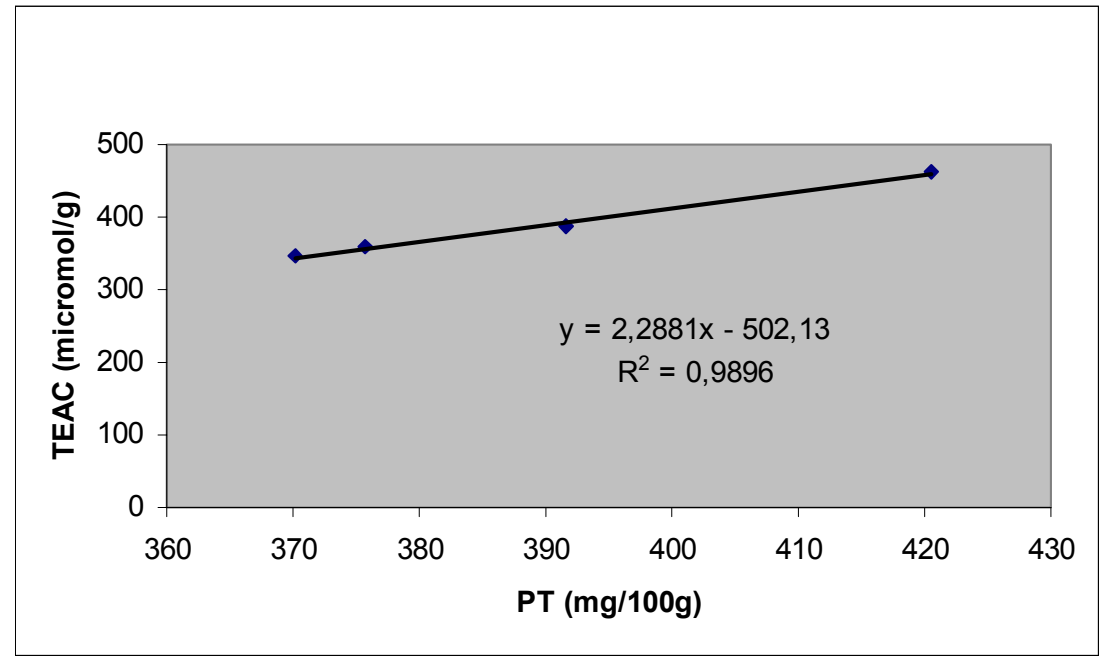

Figura 4. Correlação entre a atividade antioxidante equivalente ao Trolox (TEAC) e conteúdo de polifenóis totais (PT) dos extratos obtidos da uva PINOT GRIS.

Sun et al. (2002) e Chinnici et al. (2004) encontraram uma correlação positiva entre a atividade antioxidante e o índice de polifenóis totais. A atividade antioxidante da maçã foi correlacionada não somente à concentração de polifenóis totais, mas também à composição dos mesmos.

\section{Conclusão}

Os dados experimentais sugerem como fonte alternativa de compostos fenólicos, a utilização das biomassas residuais da indústria vitivinícola, mais especificamente, com relação às duas variedades de uvas estudadas (COUDERC 13 e PINOT GRIS). Devido às suas propriedades antioxidantes, abrem espaço para uma série de perspectivas de sua exploração, principalmente na indústria de fitoterápicos e de complementos alimentares.

\section{Referências}

AUSTRALIAN OFFICIAL ANALYTICAL CHEMISTS INTERNATIONAL - AOAC. Official methods of analysis of the Aoac International. 17. ed. Arlington, 2002. Cap. 37. 
ARNOUS, A.; MAKRIS, D.; KEFALAS, P. Effect of principal polyphenolic components in relation to antioxidant characteristics of aged red wines. Journal of Agricultural and Food Chemistry, Columbus, v. 49, n. 12, p. 5736-5742, 2002.

ARUOMA, O.I. Free radicals, oxidative stress and antioxidants in human health and disease. Journal of American Oil Chemists Society, Champaign, v. 75, n. 2, p. 199-212, 1998.

BITSCH, R. Pflanzenphenole und ihre gesundheitliche Wirkung. Naturwissenschaftliche Rundschau, Stuttgart, v. 49, n. 2, p. 47-51, 1996.

BLOCK, G. The data support a role for antioxidants in reducing cancer risk. Nutrition Reviews, v. 50, n. 7, p. 207213, 1992.

BUDINI, R.; TONELLI, D.; GIROTTI, S. Analysis of total phenols using the Prussian blue method, Journal of Agricultural and Food Chemistry, Columbus, v. 28, n6, p. 1236-1238, 1980.

CAMPOS, L. Obtenção de extratos de bagaço de uva Cabernet Sauvingnon (Vitis vinifera): parâmetros de processo e modelagem matemática. 2005. Tese (Mestrado em Engenharia de Alimentos) - Departamento de Engenharia de Alimentos, Universidade Federal de Santa Catarina, Florianópolis.

CHINNICI, F.; BENDINI, A.; GAIANI, A.; RIPONI, C. Radical scavenging activities of peels and pulps from $\mathrm{cv}$. golden delicious apples as related to their phenolic composition. Journal of Agricultural and Food Chemistry, Columbus, 52, n.15, p. 4684-4689, 2004.

EMPRESA BRASILEIRA DE PESQUISA AGROPECUARIA - EMBRAPA. Centro nacional de pesquisa em uva e vinho. Dados da viticultura: banco de dados de uva, vinho e derivados. Disponível em: <www.cnpuv.embrapa.br> Acesso em: 02 jan 2006.

FRANKEL, E.N.; WATERHOUSE, A.L; TEISSEDRE, P.L., Principle phenolic phytochemicals in selected California wines and their antioxidant activity in inhibiting oxidation of human low-density lipoprotein. Journal of Agriculture and Food Chemistry, Columbus, v. 43, n.4, p. 890-894, 1995.

HERRMANN, K. Pflanzlichen Lebensmitteln vorkommende Flavonoide als Antioxidantien. Gordian, Hamburg, v. 93, n.7-8, p. 108-111, 1994.

ITO, N.; FUKUSHIMA, S.; HASEGAWA, A.; SHIBATA, $\mathrm{M}$; OGISO, T. Carcinogenicity of butylated hydroxyanisole in F344 rats. Journal of the National Cancer Institute, Cary, v. 70, n.2, p. 343-347, 1983.

KÄHKÖNEN, M.P., HOPIA, A.I., VUORELA, H.J., RAUHA, J.P., PIHLAJA, K., KUJALA, T.S. AND
HEINONEN, M. Antioxidant activity of plant extracts containing phenolic compounds. Journal Agricultural and Food Chemistry, Washington, v.47, n.10, p. 3954 3962,1999 .

KIM, D, 0; JEONG, S. W.; LEE, C.Y. Antioxidant capacity of phenolic phytochemicals from various cultivars of plums. Food Chemistry, Amsterdam, v. 81, n. 3, p. 321-326, 2003.

KOVAC, V.; PEKIC, B. Proanthocyanidols from grape and wine. Contempory Agriculture, v. 39, n. 4, p. 5-17, 1991.

LAI, L S.; CHOU, T.; CHAO, W. W. Studies on the antioxidative activities of Hsian-tsao (Mesona procumbens Hemsl) leaf gum. Journal of Agricultural and Food Chemistry, Columbus, v. 49, n. 2, p. 963-968, 2001.

LAPARRA, J.; MICHAUD, J.; MASQUELIER, J. Action of oligermeric procyanidins on vitamin $C$ deficient guinea pig. Bulletin de la Societe de Pharmacie de Bordeaux, Bordeaux, v. 118, n. 1-2, p. 7_13, 1979.

LEE S. C.; KIM J. H.; JEONG S. M.; KIM D. R.; HA J U.; NAM K.C. Effect of far-infrared radiation on the antioxidant activity of rice hulls. Journal of Agricultural and Food Chemistry, Columbus, v. 51, n. 15, p. 4400-4403, 2003.

LLOBERA, A; CAÑELLAS, J. Dietary fibre content and antioxidant activity of mano negro red grape (Vitis vinifera): pomace and stem. Food Chemistry, Amsterdan, v. 101, n.2, p. 659-666, 2006.

MAZZA, G. Anthocyanins in grapes and grape products. Criterial Review of Food Science and Nutrition, Philadelphia, v. 35, n. 4, p. 341-371, 1995.

MELO, E. A.; GUERRA, N. B. Ação antioxidante de compostos fenólicos naturalmente presentes em alimentos. Boletim da Sociedade Brasileira de Ciências e Tecnologia de Alimentos, Campinas, v. 36, n. 1, p. 1-11, 2002.

NEGRO, C.; TOMMASI, L; MICELI, A. Phenolic compound as and antioxidant activity from red grape marc extracts. Bioresource Technology, Essex, v. 87, n. 1, p. 41-44, 2003.

PELLEGRINI, R.E.; PROTEGGENTE, N.; PANNALA, YANG, A. M.; RICE-EVANS, C. Antioxidant activity applying an improved ABTS radical cation decolorization assay. Free Radical Biology and Medicine, Orland, v. 26, n. 9-10, p. 1231-1237, 1999.

PRIOR, R. L.; CAO, G.; MARTIN, A.; LISCHNER, N.; EHLENFELDT, M.; KALT, W.; KREWER, G.; MAINLAND, C. M. Antioxidant capacity as influenced by total phenolics and anthocyanin content, maturity, and variety of Vaccinum species. Journal of Agricultural and Food Chemistry, Columbus, v.46, n. 7, p. 2686-2693, 1998. 
RE, R.; PELLEGRINI, N.; PROTEGGENTE, A.; PANNALA, A.; YANG, M.; RICE-EVANS, C. Antioxidant activity applying an improved ABTS radical cation decolorization assay. Free Radical Biology and Medicine, Orland, v. 26, n. 9-10, p. 1231-1237, 1999.

RENAUD, S.; DE LORGERIL, M. Wine, alcohol, platelets, and the French paradox for coronary heart disease. The Lancet, London, v. 339, n. 8808, p. 1523-1526, 1992.

RHODES, M. J. C. Physiologically-active compounds in plant food: an overview. Proceedings of the Nutrition Society, Cambridge, v. 55, n. 1B, p. 371-384, 1996.

RICE-EVANS, C. A.; MILLER, N. J.; PAGANGA, G. Structure-antioxidant activity relationships of flavonoids and phenolic acids. Free Radical Biology and Medicine, Orland, v. 20, n. 7, p. 933-956, 1996.

SANT'ANA, L. S.; MANCINI FILHO, J. Ação Antioxidante de extratos de alecrim (Rosmarinus officinalis L.) em filés de peixe da espécie pacu (Piaractus mesopotamicus Holmberg). Revista Brasileira de plantas medicinais, Botucatu, v. 2, n. 1, p. 27-31, 1999.

SUN, J.; CHU, Y.F.; WU, X.; LIU, R. H. Antioxidant and antiproliferative activities of common fruits. Journal of Agricultural and Food Chemistry, Columbus, v. 50, n. 25, p. 7449-7454, 2002.
THOMAS, J. H; DRAKE, J. M; PADDOCK, J. R; CONKLIN, S.; JOHNSON, J.; SELISKAR, C. Characterization of ABTS at a polymer-modified electrode. Electroanalysis, cidade, v. 16, n. 7, p. 547-555, 2004.

TORRES, J. B.; VARELA, B.; GARCIA, M. T.; CARILLA, J.; MATITO, C.; CENTELLES, J. J.; CASCANTE, M.; SORT, X; BOBET, R. Valorization of grape (Vitis vinifera) byproducts. Antioxidant and biological properties of polyphenolic fractions differing in procyanidin composition and flavonol content. Journal of Agricultural and Food Chemistry, Columbus, v. 50, n. 26, p. 7548-7555, 2002.

VALDERRAMA, P.; MARANGONI, F.; CLEMENTE, E. Efeito do tratamento térmico sobre a atividade de peroxidase (POD) e polifenoloxidase (PPO) em maçã (Mallus comunis). Ciência e Tecnologia de Alimentos, Campinas, v. 21, n. 3, p. 321-325, 2001.

VELIOGLU, Y. S.; MAZZA, G.; GAO, L.; OOMAH, B. D. Antioxidant activity and total phenolics in selected fruits, vegetables, and grain products. Journal of Agricultural and Food Chemistry, Columbus, v. 46, n. 10, p. 4113-4117, 1998.

WANG, S. Y.; LIN, H., S. Antioxidant activity in fruits and leaves of blackberry, raspberry, and strawberry varies with cultivar and developmental stage. Journal of Agricultural and Food Chemistry, Columbus, v. 48, n. 2, p. 140-146, 2000. 
